\title{
Preparation of Poly O-Toluidine/Titanium Dioxide by In situ Chemical Oxidation Polymerization and Application for Solar Cell.
}

\author{
Hayder A. Hasan", Khalid I. Ajeel* \\ Physics department, College of education, University of Basrah, Iraq. \\ E-mail of the corresponding author: hayder.physics@yahoo.com
}

\begin{abstract}
Various treatments on the PEDOT:PSS films were carried out to investigate it's influence on the conductivity, morphology, transmittance and the corresponding impact of the performance of the organic photovoltaic devices based on the PCPDTBT:PCBM and P3HT:PCBM blends. These processing including doping PEDOT:PSS with DMF and ME solvents and exposing these films to the vapor of DMF and ME solvents, separately. A considerable enhancement of the conductivity and transmittance of PEDOT:PSS was observed after doping solvent into the PEDOT;PSS solution followed by solvent treatment through exposing these films to solvents environment. The best organic PV doped devices based on either PCPDTBT:PCBM or based on P3HT:PCBM with power conversion efficiency were $2.93 \%$ compared to $1.87 \%$ for the pristine PV devices or $2.79 \%$ compared to $1.77 \%$ for the pristine devices, respectively. The conductivity improvement was highly influenced by solvent treatment.
\end{abstract}

Key words: P3HT:PCBM; PCPDTBT:PCBM; Organic solar cells; PEDOT:PSS; solvent treatment.

\section{Introduction}

Poly(ethylene-3,4-dioxythiophene): poly(styrenesulfonic acid) (PEDOT:PSS) is widely used in molecular electronic devices as a transparent anode with relatively high work function and as a smoothing coating layer of rough inorganic conducting surfaces (normally indium-tin oxides)[1]. PEDOT:PSS is well-known as one of the most favorable conductive polymers used as a hole transporting layer, due to its 
simple processing, high transparency and stability. PEDOT:PSS has been widely utilized as an anode interfacial layer to enhance the anode contact as well as to increase the holes transporting in the polymer solar cells [2]. It has been found that the electrical conductivity of PEDOT:PSS is significantly increased by the addition of a small amount of organic solvents [1,3,4], adding metal salts to the PEDOT:PSS [1] and adding acids [5]. The mechanism of this increase, however, is still uncertain. Furthermore, it has been reported in the literature that the annealing process, which is normally applied as a necessary step in the device fabrication, creates two conflicting effects on the film conductivity, leading to change the PEDOT:PSS surface ratio [6] or screening effect of the solvent, respectively [7]. The method of the film preparation from the mixture of the aqueous dispersion of the polymer and an organic additive frequently leads to more rough film morphology as compared to the films prepared from the pure aqueous dispersions [1,7]. PEDOT:PSS is wellknown as mixture of conjugated conducting polymer PEDOT blended with nonconjugated water-soluble PSS polymer [8]. Several studies have revealed that the treatment of aqueous PEDOT:PSS solution by different solvents such as methanol, ethanol, isopropanol, glycerol, ethylene glycol, diethylene glycol and D-sorbitol is significantly enhance the conductivity of PEDOT:PSS, this enhancement was attributed to the removing the PSS component within the PEDOT:PSS films to some extent [9-13]. The most conductive formulation of PEDOT:PSS (CLEVIOS PH 1000 ) is used in OSC device by Vosgueritchian et.al. [14], they have claimed that the incorporating fluorosurfactant as an additive to PEDOT:PSS is resulted in $35 \%$ improvement in sheet resistance (Rs) compared to untreated PEDOT:PSS thin films. Moreover, large area, highly conductive films prepared by spray-coating of EG/PH1000 PEDOT:PSS doped with graphene with excellent mechanical properties and used as ultrathin electrode in OSC devices [15]. Another type of PEDOT:PSS conductive ink is PEDOT-PSS Al4083 doped with Au nanoparticles is also used in OSC device [16]. The increasing of Au nanoparticles in PEDOT:PSS improves the efficiency in about $13 \%$ to achieve $3.51 \%$. The latter improvement is attributed to the increase in FF and $J_{s c}$, from 0.58 to 0.62 and $8.5 \mathrm{~mA} . \mathrm{cm}^{-2}$ to $8.94 \mathrm{~mA} . \mathrm{cm}^{-2}$, 
respectively. Further increase in the concentration of Au nanoparticles has shown a negative effect on the device performance [17]. Using spin coating method, PEDOT:PSS can easily form a transparent film with high conductivity and low sheet resistance film [18]. The combination of conductivity and morphology is critical for various applications of PEDOT:PSS thin films [1]. The latter films could be influenced several factors during the drying process including the fraction of solid content, particle size, proportion of PSS and the solution viscosity which could lead to different morphological and electrical properties [19]. Moreover, the solar cell performance is mainly determined by the amount known as the power conversion efficiency (PCE) which depends on several parameters such as light harvesting of the active layer, excitons generation, diffusion, separation, transportation and collection by the electrodes [20]. Organic solar cell (OSC) devices, fabricated from the blends of Poly[[9-(1-octylnonyl)-9H-carbazole-2,7-diyl]-2,5-thiophenediyl2,1,3-benzothiadiazole-4,7-diyl-2,5-thiophenediyl]:[6,6]-phenyl-C71-butyric acid methyl esters (PCDTBT:PC ${ }_{71} B M$ ) bulk heterojunction systems has relatively good photovoltaic (PV) properties compared to other bulk heterojunction OSCs [21]. In this work, the effects of treated PEDOT:PSS with methoxyethanol (ME) and Dimethylformamide (DMF) have been carried out. Usually, PEDOT:PSS treated with either solvent additives or solvent vapor treatment. In the current study, two strategies were employed the first is doping PEDOT:PSS with either ME or DMF and the second is treated the doped PEDOT:PSS layer with solvent treatment (using the same solvent, i.e. using DMF solvents treatment for DMF-doped PEDOT:PSS layer). This treatment has direct impact on the electrical conductivity, morphology, optical other properties of PEDOT:PSS layer and therefore on the organic solar cells performance.

\section{Experiment}

\subsection{Materials and chemicals}

Poly (3,4ethylenedioxythiophene):poly(styrenesulfonate) (PEDOT:PSS) layer (Sigma-Aldrich $\mathrm{PH} 1000)$, indium doped tin oxide (ITO) (80 nm in thickness and 25 
$\Omega / \mathrm{sq}$ sheet resistance), methoxyethanol (ME) , Dimethylformamide (DMF), chlorobenzene (CB), Poly[9-(1-octylnonyl)-9H-carbazole-2,7-diyl]-2,5thiophenediyl-2,1,3-benzothiadiazole- 4,7-diyl-2,5-thiophenediyl (PCDTBT), Poly (3-hexathiophine-2,5-diyl) (P3HT) and [6,6]-Phenyl C61butyric acid methyl ester (PCBM), were purchased from Sigma Aldrich.

\subsection{Doping PEDOT:PSS with DMF and ME}

PEDOT:PSS aqueous solution (PH1000) was used as a hole transport layer in organic solar cell. Two steps where used here to modify the PEDOT:PSS layer, these are, firstly, $0.5 \mathrm{ml}$ of ME and DMF were added into $10 \mathrm{ml}$ PEDOT:PSS as additives [22, 23], separately. The solution mixture was stirred vigorously for 5 hours, after that it was filtered using a $0.45 \mu \mathrm{m}$ PVDF filter. The filtered PEDOT:PSS was then deposited by spin coating at $2000 \mathrm{rpm}$ for $30 \mathrm{sec}$ on the top of ITO substrates. Spectroscopic ellipsometry has been used to measure the thin film thickness, the latter is found to be about 100nm [24] with small variation between the studied thin films. ITO coated glass was pre-cleaned with water, ethanol and methanol in ultrasonic bath for $10 \mathrm{~min}$ each, respectively. Pristine and doped PEDOT:PSS films were annealed at $150^{\circ} \mathrm{C}$ for $10 \mathrm{~min}$. Secondly, the dopedPEDOT:PSS layer where subjected to further solvent treatment for $2 \mathrm{~h}$ each; DMFdoped PEDOT:PSS layer was subjected to DMF solvent treatment as well as the ME-doped PEDOT:PSS and were annealed after the treatment at $150^{\circ} \mathrm{C}$ for 10 $\min$.

\subsection{Sample preparation}

P3HT:PCBM (in 1:1 ratio) as active layers and PCDTBT:PCBM (in 1:4 ratio) were dissolved in chlorobenzene, separately, and stirred overnight at $70^{\circ} \mathrm{C}$ to obtain the organic ink. The active layers were spin coated on the top of PEDOT:PSS layers inside a nitrogen-filled glove box followed by annealing inside the glove box at 120 ${ }^{\circ} \mathrm{C}$ for $10 \mathrm{~min}$. A top contact of aluminum (Al) was evaporated with a thickness of about $100 \mathrm{~nm}$, at the deposition rate of $0.1-0.2 \mathrm{~nm} / \mathrm{sec}$ as was monitored by a quartz crystal thickness monitor. All the devices were subjected to further heat 
treatment inside the glove box at $120{ }^{\circ} \mathrm{C}$ for $10 \mathrm{~min}$ and left to cool down for $30 \mathrm{~min}$ before measurements were carried out. Fig.1 shows the band diagram of the P3HT:PCBM and PCPDTBT:PCBM based devices under study.

\subsection{Characterization}

The optical properties for the studied layers were recorded on Varian 50-scan UVVisible spectrophotometer working in the range of $190-1100 \mathrm{~nm}$. The blend structure was investigated by multipurpose X'Pert Philips X-ray diffractometer (MPD) $(\mathrm{Cu}, \mathrm{k}=0.154 \mathrm{~nm})$. The films' morphology was determined using a Nanoscope Illa multimode atomic force microscope (Bruker-AFM). Raman characteristics have been carried out using Raman spectrometer (Renishaw, UK) with excitation wavelength $514 \mathrm{~nm}$. DC electrical characteristics in the form of current density-voltage $(\mathrm{J}-\mathrm{V})$ dependence and electrical conductivity of PEDOT:PSS layers as well as completed OSC devices were studied using 2400 computerized Sourcemeter and the photo current was generated under AM 1.5 solar simulator source of $100 \mathrm{~mW} . \mathrm{cm}^{-2}$; a shadow mask with the same active area $\left(0.07 \mathrm{~cm}^{2}\right)$ was used for the $I-V$ characterization. The fill factor $(F F)$ and the overall light to-electrical energy conversion efficiency (PCE) of the solar cell were calculated according to the following equations [25]:

$$
\begin{aligned}
& \text { PCE }(\%)=\frac{I_{\max } \mathrm{v}_{\max }}{\mathrm{P}_{\text {in }}} \\
& \text { FF }=\frac{I_{\max } \mathrm{v}_{\max }}{I_{\mathrm{gsc}} \mathrm{v}_{\text {oc }}}
\end{aligned}
$$

where $J_{s c}$ is the short-circuit current density $\left(m A \cdot \mathrm{cm}^{-2}\right), V_{o c}$ is the open-circuit voltage $(\mathrm{V}), \mathrm{P}_{\text {in }}$ is the incident light power and $\mathrm{J}_{\max }\left(\mathrm{mA} \cdot \mathrm{cm}^{-2}\right)$ and $\mathrm{V}_{\max }(\mathrm{V})$ are the current density and voltage at the point of maximum power output in the $\mathrm{J}-\mathrm{V}$ curves, respectively. Furthermore, the electrical conductivity was determined using DropSens interdigitated Platinum electrodes (IDEs). The IDE can be used to measure the surface conductivity $(\sigma)$ of the samples from the following relationship [1]:

$$
\sigma=\frac{\mathrm{I}}{\mathrm{v}} \frac{\mathrm{n}}{\mathrm{WtL}}
$$


where, $t$ is the thickness of the film, $W$ is the distance between the fingers $(6.67 \mathrm{~mm}), \mathrm{n}$ is the number of fingers $(500)$, and $(L)$ is the distance between electrodes $(5 \mu \mathrm{m})$.

\section{Results and discussion}

\subsection{Effect of treatment on the PEDOT:PSS properties:}

Fig. 2 shows the transmittance spectra of the PEDOT:PSS based layer before and after treatment. It is clear that the transmittance intensity has slightly increased after treating the PEDOT:PSS layer. The pure PEDOT:PSS layer has demonstrated a maximum transmittance of about $91 \%$ around $450 \mathrm{~nm}$. Doping the PEDOT:PSS with ME and DMF has resulted in increasing the transmittance spectra slightly as demonstrated in Fig.2. The improved transparency of PEDOT:PSS layer is found to be beneficial for OSCs electronics [26]. Further improvement in the transparency of the PEDOT:PSS thin films has been observed after further solvent treatment. Generally, all the PEDOT:PSS thin films show good transmittance spectra in the range from 400 to $800 \mathrm{~nm}$ which could be attributed to the colorless nature of the PSS, the optical absorption in PEDOT:PSS mainly originates from the presence of PEDOT [27]. This intensity difference cannot be attributed to the loss of PSS from the PEDOT:PSS thin films, because all the solvents were removed from the PEDOT:PSS films by heating and the treated PEDOT:PSS films were not rinsed with any solvent. Thus, the transmittance difference should be due to a solventinduced change in the morphology of the PEDOT:PSS films [12]. Xia et.al [12] have reported a simple model using Beer-Lambert law to conclude the change in the intensities after treating PEDOT:PSS layer with solvents. They have claimed that if the PEDOT:PSS layer has different transmittance intensities based on how uniform the PEDOT:PSS covers the substrate. They have observed that PEDOT:PSS transmittance properties is increased after solvent treatment and suggested the aggregation of the PSS chains [12]. Similar observation has been recorded in here, where the PSS aggregation is confirmed by the AFM images (Fig.3). AFM images shows that the surface morphology of the PEDOT:PSS layers has influenced by the solvents additives, the surface roughness of the pure PEDOT:PSS layer is found to 
be $0.98 \mathrm{~nm}$. This has slightly increased after doping with ME to $1.03 \mathrm{~nm}$. Doping PEDOT:PSS with DMF has shown an increase in the surface roughness to $1.18 \mathrm{~nm}$; this increase has confirmed the aggregation of PSS. Further treatment has resulted in further PSS separation and rough surface has observed; ME solvent treated ME doped PEDOT:PSS layer has demonstrated rougher surface $2.1 \mathrm{~nm}$ and DMF solvent treated DMF doped PEDOT:PSS layer has revealed surface roughness of 2 $\mathrm{nm}$. The increase in the surface roughness likely increase the contact area between the PEDOT:PSS and the active layer, improving hole extraction to the anode [1]. Furthermore, the phase separation occurs between PEDOT and PSS sites after treatment is also favorable for increasing the electrical conductivity of the PEDOT:PSS layers. The electrical conductivity increases with after doping PEDOT:PSS with ME from $10 \pm 2 \mathrm{~S} / \mathrm{cm}$ to $163 \pm 5 \mathrm{~S} / \mathrm{cm}$ as shown in Fig. 4 . The conductivity has further increased after subjected the ME doped PEDOT:PSS layer to $\mathrm{ME}$ solvent treatment for several hours resulting in more site separation between PEDOT and PSS and significantly increase the electrical conductivity to $178 \pm 8 \mathrm{~S} / \mathrm{cm}$. Similar results have been observed using DMF both as doping material $(197 \pm S / \mathrm{cm})$ and as solvent treatment $(202 \pm 8 \mathrm{~S} / \mathrm{cm})$. The increased conductivity in the presence of solvent is believed to arise from the rearrangement of the PEDOT:PSS morphology leading to better connections between the conducting PEDOT chains, proposed that the conductivity enhancement is strongly dependent on the chemical structure of the solvents. Cruz-Cruz et.al have shown that the use of DMSO solvents has resulted in increasing the electrical conductivity and this increase was attributed to the phase separation of the excess PSS. Therefore the insulation of conducting PEDOT:PSS domains is reduced [28]. Higher conductivity of PEDOT:PSS can reduce the interface contact barrier, and improve photo induced carrier transporting resulting in increasing the short circuit current density [29]. In order to investigate the deformation in PEDOT:PSS thin films after treatment, Raman spectroscopy has been carried out and the results are shown in Fig.5(A and B). Clear differences between the Raman spectra of the pure and the additive treated PEDOT:PSS films in the range from 1400 to $1600 \mathrm{~cm}^{-1}$ has been observed. 
The vibrational modes $(\mathrm{C}-\mathrm{C})$ of PEDOT are located at $1453 \mathrm{~cm}^{-1}$; this has shown a clear shift to $1448 \mathrm{~cm}^{-1}, 1447 \mathrm{~cm}^{-1}, 1446 \mathrm{~cm}^{-1}$ and $1444 \mathrm{~cm}^{-1}$ after doping with ME, DMF, ME with ME solvent treatment and DMF with DMF solvent treatment, respectively. Another vibrational mode of PEDOT:PSS assigned to $\mathrm{C}-\mathrm{C}$ stretching is also observed around $1400 \mathrm{~cm}^{-1}$ and around $1520 \mathrm{~cm}^{-1}$ while the vibrational mode of PSS is located around $1550 \mathrm{~cm}^{-1}$ [30-32]. The Raman shift in the PEDOT vibrational mode indicating a conformation of the PEDOT changes from coil conformation to linear conformation after the treatment [33]. The partial removal of PSS is observed by the reduction in intensity of the Raman fingerprints [34]. If the polymer chains have linear orientation where the neighboring thiophene rings are mostly oriented in the same plane, therefore the conjugated $\pi$-electrons should be delocalized over the whole polymer chain resulting in higher charge-carrier mobility as compared to coil structure [33]. Therefore, the interaction between PEDOT chains becomes stronger and can enhance the intra-chain and interchain chargecarrier mobility, so that the conductivity gets increased as discussed before.

\subsection{Solar cell characteristics}

The effects of PEDOT:PSS with and without treatment on the photovoltaic properties have been carried out using two different blends with two different conjugated polymers, P3HT:PCBM and PCPDTBT:PCBM as shown in Fig.6 (A and $B)$. It I clear that the short circuit current density $\left(\mathrm{J}_{\mathrm{SC}}\right)$ has increased noticeably after doping the PEDOT:PSS layer with ME and DMF and after solvents treatment as shown in table 1 and table 2. Fig.6 (A) is demonstrated the effects of these treatment on the PCPDTBT:PCBM-based solar cells, $\mathrm{J}_{\mathrm{SC}}$ has increased from $7.85 \mathrm{~mA} . \mathrm{cm}^{-2}$ in the device using pure PEDOT:PSS layer to $11.17 \mathrm{~mA} . \mathrm{cm}^{-2}$ and 10.65mA. $\mathrm{cm}^{-2}$ for PEDOT:PSS layers doped with ME and DMF, respectively. In case of P3HT:PCBM based solar cells, similar behavior has been observed and $\mathrm{J}_{\mathrm{SC}}$ has increased from $4.59 \mathrm{~mA} . \mathrm{cm}^{-2}$ in the device using pure PEDOT:PSS layer to 5.38mA. $\mathrm{cm}^{-2}$ and 5.88mA.cm ${ }^{-2}$ for PEDOT:PSS layers doped with ME and DMF, respectively. Further increase in $J_{S C}$ is also has also detected after subjected the 
treated PEDOT:PSS layers to solvent treatment, the results are shown in tables 1 and 2. The increase in the short circuit current density is thought to be due to the enhance the electrical conductivity of the PEDOT:PSS layers after doping and therefore reduce the interface contact barrier, and improving the photo induced carrier transporting resulting in increasing the short circuit current density [29]. Moreover, the increase in the surface roughness likely increase the contact area between the PEDOT:PSS and the active layer, improving hole extraction to the anode [1]. Also, the rough surface of PEDOT:PSS layer after treatment is more likely increase the light scattering entering the active layer [35], consequently it might also contribute in longer light propagation path within the active layer [24]. Fig. 7 shows an assumption of the scattered light from the ITO/PEDOT:PSS rough surface. Increasing $\mathrm{J}_{\mathrm{sc}}$ in the studied devices has been confirmed by measured the incident photon-to-current collection efficiency (IPCE) spectra of both P3HT:PCBM and PCPDTBT based devices using PEDOT:PSS based and without treatment as buffer layer as shown in Fig.8. Each device shows the known spectral response of its bulk heterojunction blend. The P3HT:PCBM based devices has demonstrated a maximum IPCE about $40 \%$ at around the wavelengths of 500-550nm [36]. While the PCPDTBT:PCBM based device has two dominant peaks the first one about $30 \%$ at around $500 \mathrm{~nm}$ and the second is about $40 \%$ around the wavelength $650 \mathrm{~nm}$ [37]. Furthermore, no clear change in the open circuit voltage $\left(V_{O C}\right)$ has been observed for all the studied devices. Basically $V_{O C}$ is determined by the following equation [38]:

$$
\mathrm{V}_{\mathrm{OC}}=\left[\mathrm{LUMO}_{\text {acceptor }}-\mathrm{HOMO}_{\text {donor }}\right]-\cdot \cdot \mathrm{r}
$$

Higher $V_{O C}$ has been recorded for the PCPDTBT:PCBM based devices within the range of $0.68 \pm 0.01 \mathrm{~V}$ and $0.64 \pm 0.01 \mathrm{~V}$ for the $\mathrm{P} 3 \mathrm{HT}$ :PCBM based devices as shown in Table 1 and Table 2. No clear change in FF values has been observed for PCPDTBT:PCBM based devices with FF of $0.35 \pm 0.01$. On the other hand, P3HT:PCBM based devices have demonstrated obvious increase in FF from 0.61 in pure PEDOT:PSS based device (for P3HT:PCBM blend) to 0.66 in the device with PDOT:PSS doped with DMF and DMF solvent treatment (for P3HT:PCBM blend). 
This increase could be attributed to the enhancement in the series resistance after doping the PEDOT:PSS layer (see Table 1 and Table 2), this enhancement is correlated to the increase in the electrical conductivity of the PEDOT:PSS layer as mentioned before. However, PCPDTBT:PCBM based device have shown low FF in general due to the low crystallinity of PCPDTBT material which has a bulk side chain [39]. The power conversion efficiency (PCE) for the studied devices are shown in Table 1 and Table 2, for the pure PEDOT:PSS, PCE of $1.78 \%$ has been recorded for PCPDTBT:PCBM based device. This PCE has increase to $2.93 \%$ in the device with PDOT:PSS doped with DMF and DMF solvent treatment. This increase has been mainly attributed to the increase in the short circuit current density [40]. In case of P3HT:PCBM based devices, similar behavior has been observed where the device with the untreated PEDOT:PSS has demonstrated a PCE of $1.77 \%$ and this has increase to $2.79 \%$ in the device with PDOT:PSS doped with DMF and DMF solvent treatment. This enhancement has been ascribed to the increase in the short circuit current density [24,40] as well as the obvious enhancement in FF.

\section{Conclusion:}

In summary, the enhancement in the PEDOT:PSS properties has direct impact on the performance of the studied organic solar cells. The PEDOT:PSS treatments were carried out in two ways, firstly doping with ME and DMF and secondly solvent treatment with the same solvent. The organic solar cells based on PCPDTBT:PCBM blend as an active layer has demonstrated slight improvement in the performance of the devices compared to those which fabricated using P3HT:PCBM as an active layer. According to this study, it has been found that the conductivity of the PEDOT:PSS play a key role of the improvements of the organic devices.

\section{Acknowledgment:}

The authors are gratefully acknowledge the support from advances polymer lab, Physics Department, College of Science University of Babylon, University of Missan, and Sheffield Hallam University 


\section{References:}

[1] Kadem, B., Cranton, W. and Hassan, A., Metal salt modified PEDOT: PSS as anode buffer layer and its effect on power conversion efficiency of organic solar cells. Organic Electronics, 24(2015): 73-79.

[2] Yun, J.M., Yeo, J.S., Kim, J., Jeong, H.G., Kim, D.Y., Noh, Y.J., Kim, S.S., $\mathrm{Ku}$, B.C. and $\mathrm{Na}$, S.I., Solution-Processable Reduced Graphene Oxide as a Novel Alternative to PEDOT: PSS Hole Transport Layers for Highly Efficient and Stable Polymer Solar Cells. Advanced Materials, 23(2011):4923-4928.

[3] Kim, Y.H., Sachse, C., Machala, M.L., May, C., Müller-Meskamp, L. and Leo, K., Highly conductive PEDOT: PSS electrode with optimized solvent and thermal post-treatment for ITO-free organic solar cells. Advanced Functional Materials, 21(2011):1076-1081.

[4] Zhang, S., Fan, Z., Wang, X., Zhang, Z. and Ouyang, J., Enhancement of the thermoelectric properties of PEDOT: PSS via one-step treatment with cosolvents or their solutions of organic salts. Journal of Materials Chemistry A, 6(2018):7080-7087.

[5] Wu, Z., Yu, Z., Yu, H., Huang, X. and Chen, M., Effect of trifluoroacetic acid treatment of PEDOT: PSS layers on the performance and stability of organic solar cells. Journal of Materials Science: Materials in Electronics, 29(2018):6607-6618.

[6] Greczynski, G., Kugler, T. and Salaneck, W.R., Characterization of the PEDOT-PSS system by means of $\mathrm{X}$-ray and ultraviolet photoelectron spectroscopy. Thin Solid Films, 354(1999):129-135.

[7] Brabec, C.J., Dyakonov, V., Parisi, J. and Sariciftci, N.S. eds., 2013. Organic photovoltaics: concepts and realization (Vol. 60). Springer Science \& Business Media.

[8] Groenendaal, L., Jonas, F., Freitag, D., Pielartzik, H. and Reynolds, J.R., Poly (3, 4-ethylenedioxythiophene) and its derivatives: past, present, and future. Advanced materials, 12(2000):481-494. 
[9] Zhang, F., Johansson, M., Andersson, M.R., Hummelen, J.C. and Inganäs, O., Polymer photovoltaic cells with conducting polymer anodes. Advanced Materials, 14(2002):662-665.

[10] Hsiao, Y.S., Whang, W.T., Chen, C.P. and Chen, Y.C., High-conductivity poly (3, 4-ethylenedioxythiophene): poly (styrene sulfonate) film for use in ITO-free polymer solar cells. Journal of Materials Chemistry, 18(2008):5948-5955.

[11] Kim, J.R., Jung, J.H., Shin, W.S., So, W.W. and Moon, S.J., Efficient TCOfree organic solar cells with modified poly (3,4-ethylenedioxythiophene): poly (styrenesulfonate) anodes. Journal of nanoscience and nanotechnology, 11(2011):326-330.

[12] Xia, Y. and Ouyang, J., PEDOT: PSS films with significantly enhanced conductivities induced by preferential solvation with cosolvents and their application in polymer photovoltaic cells. Journal of Materials Chemistry, 21(2011):4927-4936.

[13] Zhang, W., Bi, X., Zhao, X., Zhao, Z., Zhu, J., Dai, S., Lu, Y. and Yang, S., Isopropanol-treated PEDOT: PSS as electron transport layer in polymer solar cells. Organic Electronics, 15(2014):3445-3451.

[14] Vosgueritchian, M., Lipomi, D.J. and Bao, Z., 2012. Highly conductive and transparent PEDOT: PSS films with a fluorosurfactant for stretchable and flexible transparent electrodes. Advanced functional materials, 22(2), pp.421428.

[15] Liu, Z., Parvez, K., Li, R., Dong, R., Feng, X. and Müllen, K., 2015. Transparent conductive electrodes from graphene/PEDOT: PSS hybrid inks for ultrathin organic photodetectors. Advanced Materials, 27(4), pp.669-675.

[16] Fung, D.D., Qiao, L., Choy, W.C., Wang, C., Wei, E.I., Xie, F. and He, S., 2011. Optical and electrical properties of efficiency enhanced polymer solar cells with $\mathrm{Au}$ nanoparticles in a PEDOT-PSS layer. Journal of Materials Chemistry, 21(41), pp.16349-16356.

[17] Fung, D.D., Qiao, L., Choy, W.C., Wang, C., Wei, E.I., Xie, F. and He, S., 2011. Optical and electrical properties of efficiency enhanced polymer solar 
cells with $\mathrm{Au}$ nanoparticles in a PEDOT-PSS layer. Journal of Materials Chemistry, 21(41), pp.16349-16356.

[18] S.K.M. Johnson, J. Birgerson, X. Crispin, G. Greczynski, W. Osikowicz, A.W. Denier van der Gon, W.R. Salaneck, M. Fahlman, The effects of solvents on the morphology and sheet resistance in poly(3,4-ethylenedioxythiophene)polystyrene sulfonic acid (PEDOT:PSS) films, Synth. Met. 139 (2003):1-10.

[19] C.S. Suchand-Sangeeth, M. Jaiswal, R. Menon, Correlation of morphology and charge transport in poly(3,4-ethylenedioxythiophene)-polystyrenesulfonic acid (PEDOT:PSS) films, J. Phys. Condens. Matter 21 (2009):072101.

[20] H.-C. Han, C.-A. Tseng, C.-Y. Du, A. Ganguly, C.-W. Chong, S.-B. Wang, C.-F. Lin, S.-H. Chang, C.-C. Su, J.-H. Lee, K.-H. Chen, L.-C. Chen, Enhancing efficiency with fluorinated interlayers in small molecule organic solar cells, J. Mater. Chem. 22 (2012) 22899.

[21] Zhang, W., Zhao, B., He, Z., Zhao, X., Wang, H., Yang, S., Wu, H. and Cao, Y., High-efficiency ITO-free polymer solar cells using highly conductive PEDOT: PSS/surfactant bilayer transparent anodes. Energy \& Environmental Science, 6(2013):1956-1964.

[22] Zhang, Y., Cui, W., Zhu, Y., Zu, F., Liao, L., Lee, S.T. and Sun, B., High efficiency hybrid PEDOT: PSS/nanostructured silicon Schottky junction solar cells by doping-free rear contact. Energy \& Environmental Science, 8(2015):297-302.

[23] Li, Z., Qin, F., Liu, T., Ge, R., Meng, W., Tong, J., Xiong, S. and Zhou, Y., Optical properties and conductivity of PEDOT: PSS films treated by polyethylenimine solution for organic solar cells. Organic Electronics, 21(2015):144-148.

[24] Hassan, A., Kadem, B. and Cranton, W., Organic solar cells: Study of combined effects of active layer nanostructure and electron and hole transport layers. Thin Solid Films, 636 (2017):760-764. 
[25] Yue Gen Tian, WU Ji Huai, XIAO Yao Ming, YE Hai Feng, LIN Jian Ming and HUANG Miao Liang. Flexible dye-sensitized solar cell based on PCBM/P3HT hetrojunction. Chin. Sci. Bull., 56 (2011):325-330.

[26] Chung-Ki Cho, Woo-JinHwang, KyoungtaeEun, Sung-HoonChoa, Seok-InNa, Han-KiKim, Mechanical flexibility of transparent PEDOT:PSS electrodes prepared by gravure printing for flexible organic solar cells, Solar Energy Materials \& Solar Cells 95 (2011):3269-3275.

[27] Kumar, V. and Wang, H., Plasmonic Au nanoparticles for enhanced broadband light absorption in inverted organic photovoltaic devices by plasma assisted physical vapour deposition. Organic Electronics, 14(2013):560-568.

[28] Cruz-Cruz, I., Reyes-Reyes, M., Aguilar-Frutis, M.A., Rodriguez, A.G. and López-Sandoval, R., Study of the effect of DMSO concentration on the thickness of the PSS insulating barrier in PEDOT: PSS thin films. Synthetic Metals, 160(2010):1501-1506.

[29] Fan, X., Fang, G.J., Qin, P.L., Cheng, F. and Zhao, X.Z., Rapid phase segregation of $\mathrm{P} 3 \mathrm{HT}$ : PCBM composites by thermal annealing for highperformance bulk-heterojunction solar cells. Applied Physics A, 105(2011):1003-1009.

[30] Garreau, S., Duvail, J.L. and Louarn, G., Spectroelectrochemical studies of poly $(3,4$-ethylenedioxythiophene) in aqueous medium. Synthetic metals, 125(2001):325-329.

[31] Han, Y.K., Chang, M.Y., Huang, W.Y., Pan, H.Y., Ho, K.S., Hsieh, T.H. and Pan, S.Y., Improved performance of polymer solar cells featuring onedimensional PEDOT nanorods in a modified buffer layer. Journal of The Electrochemical Society, 158(2011):K88-K93.

[32] Farah, A.A., Rutledge, S.A., Schaarschmidt, A., Lai, R., Freedman, J.P. and Helmy, A.S., Conductivity enhancement of poly (3, 4-ethylenedioxythiophene)poly (styrenesulfonate) films post-spincasting. Journal of Applied Physics, 112 (2011):113709. 
[33] Chaturvedi, N., Alam, F., Kumar Swami, S. and Dutta, V., Effect of electric field on the spray deposited poly (3, 4-ethylenedioxythiophene): poly (styrenesulfonate) layer and its use in organic solar cell. Journal of Applied Physics, 114(2013):184501.

[34] Chang, S.H., Chiang, C.H., Kao, F.S., Tien, C.L. and Wu, C.G., Unraveling the enhanced electrical conductivity of PEDOT: PSS thin films for ITO-free organic photovoltaics. IEEE Photonics Journal, 6(2014):1-7.

[35] Yu-Chiang Chao, Yun-Hsuan Lin, Ching-Yi Lin, Husan-De Li, Fu-Min Zhan and $\mathrm{Yu}$-Zhang Huang, Improved light trapping in polymer solar cells by light diffusion ink, J. Phys. D: Appl. Phys. 47 (2014):105102.

[36] Kadem, B.Y., Kadhim, R.G. and Banimuslem, H., Efficient P3HT: SWCNTs hybrids as hole transport layer in P3HT: PCBM organic solar cells. Journal of Materials Science: Materials in Electronics, (2018):1-9.

[37] Kim, J.Y., Lee, K., Coates, N.E., Moses, D., Nguyen, T.Q., Dante, M. and Heeger, A.J., Efficient tandem polymer solar cells fabricated by all-solution processing. Science, 317(2007):222-225.

[38] H. Li, Z.G. Zhang, Y. Li, J. Wang, Tunable open-circuit voltage in ternary organic solar cells. Appl. Phys. Lett. 101(2012):163302.

[39] Hwang, H., Lee, H., Shafian, S., Lee, W., Seok, J., Ryu, K.Y., Yeol Ryu, D. and Kim, K., 2017. Thermally Stable Bulk Heterojunction Prepared by Sequential Deposition of Nanostructured Polymer and Fullerene. Polymers, 9(9), p.456

[40] Y.C. Chao, Y.H. Lin, C.Y. Lin, F.M. Zhan, Y.Z. Huang, Improved light trapping in polymer solar cells by light diffusion ink, J. Phys. D. Appl. Phys. 47 (2014):105102. 


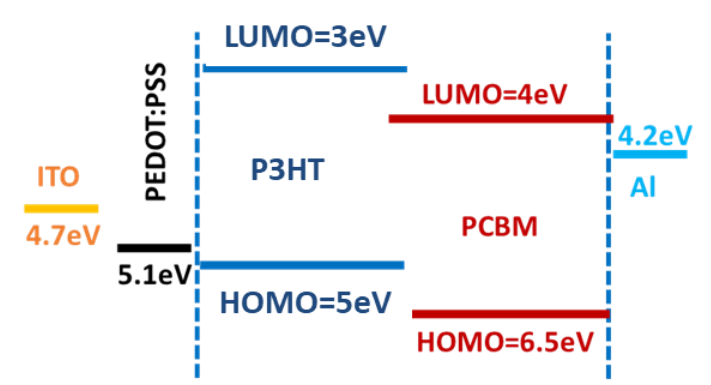

(A)

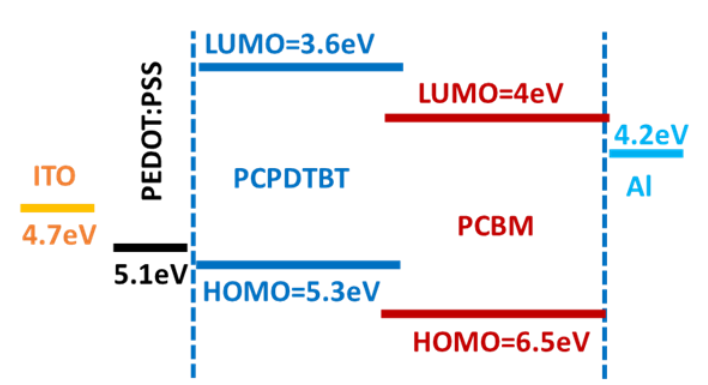

(B)

Fig.1; The band diagram of the (A) P3HT:PCBM and (B) PCPDTBT:PCBM based device under study

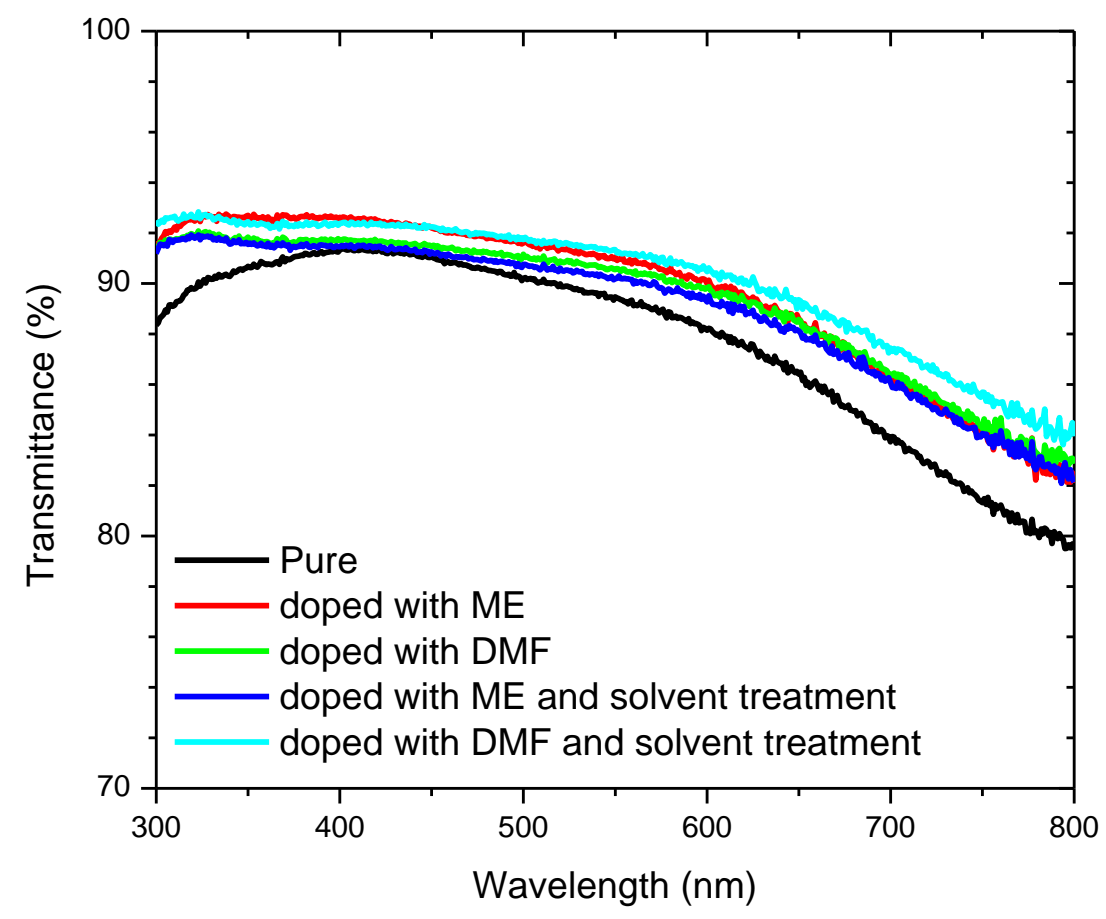

Fig.2; The transmittance spectrum of PEDOT:PSS based films, pure and treated with ME and DMF.
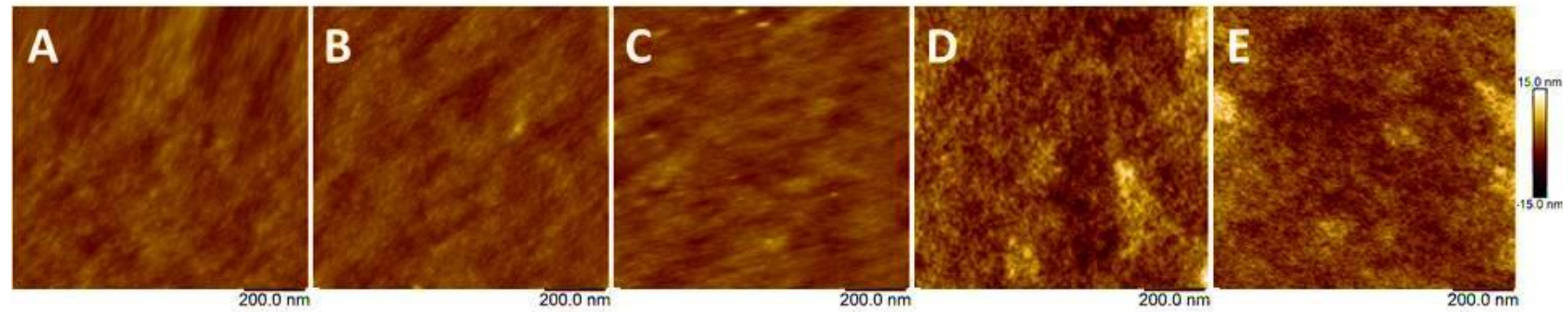
Fig.3; AFM images of (A) Pure PEDOT:PSS, (B) ME doped PEDOT:PSS, (C) DMF doped PEDOT:PSS, (D) ME doped PEDOT:PSS and ME solvent treatment and (E) DMF doped PEDOT:PSS and DMF solvent treatment

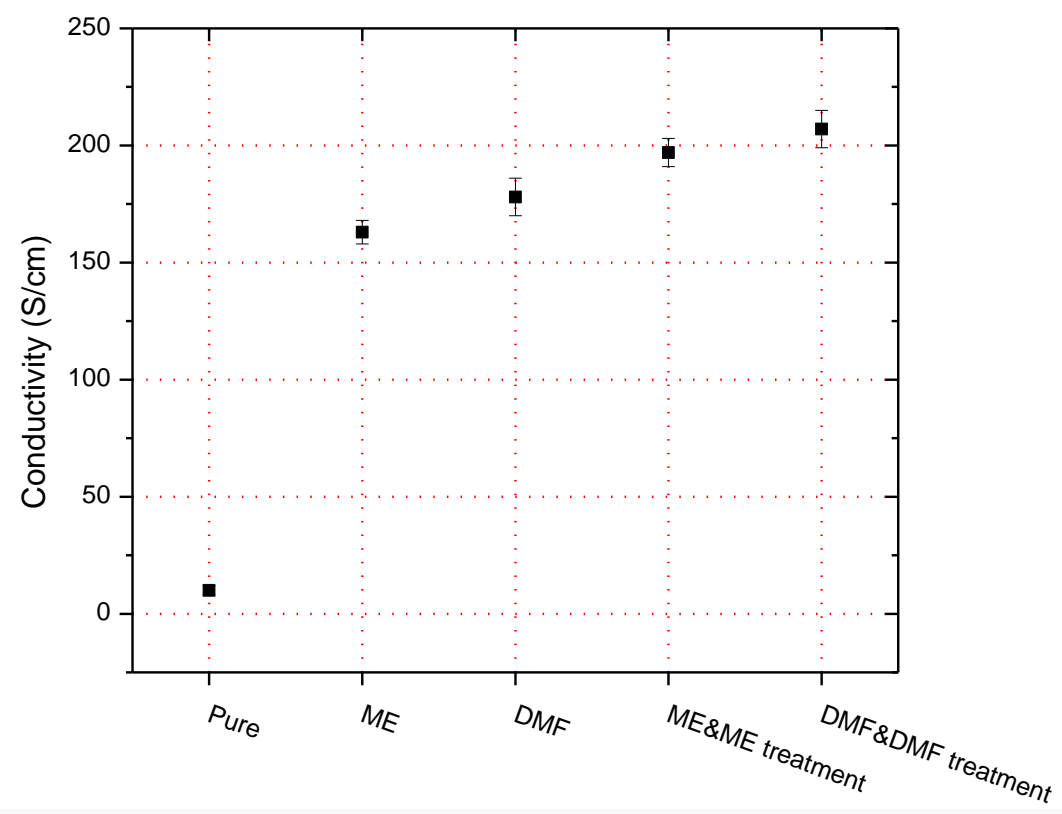

Fig.4; the electrical conductivity for the PEDOT:PSS layer with and without treatment

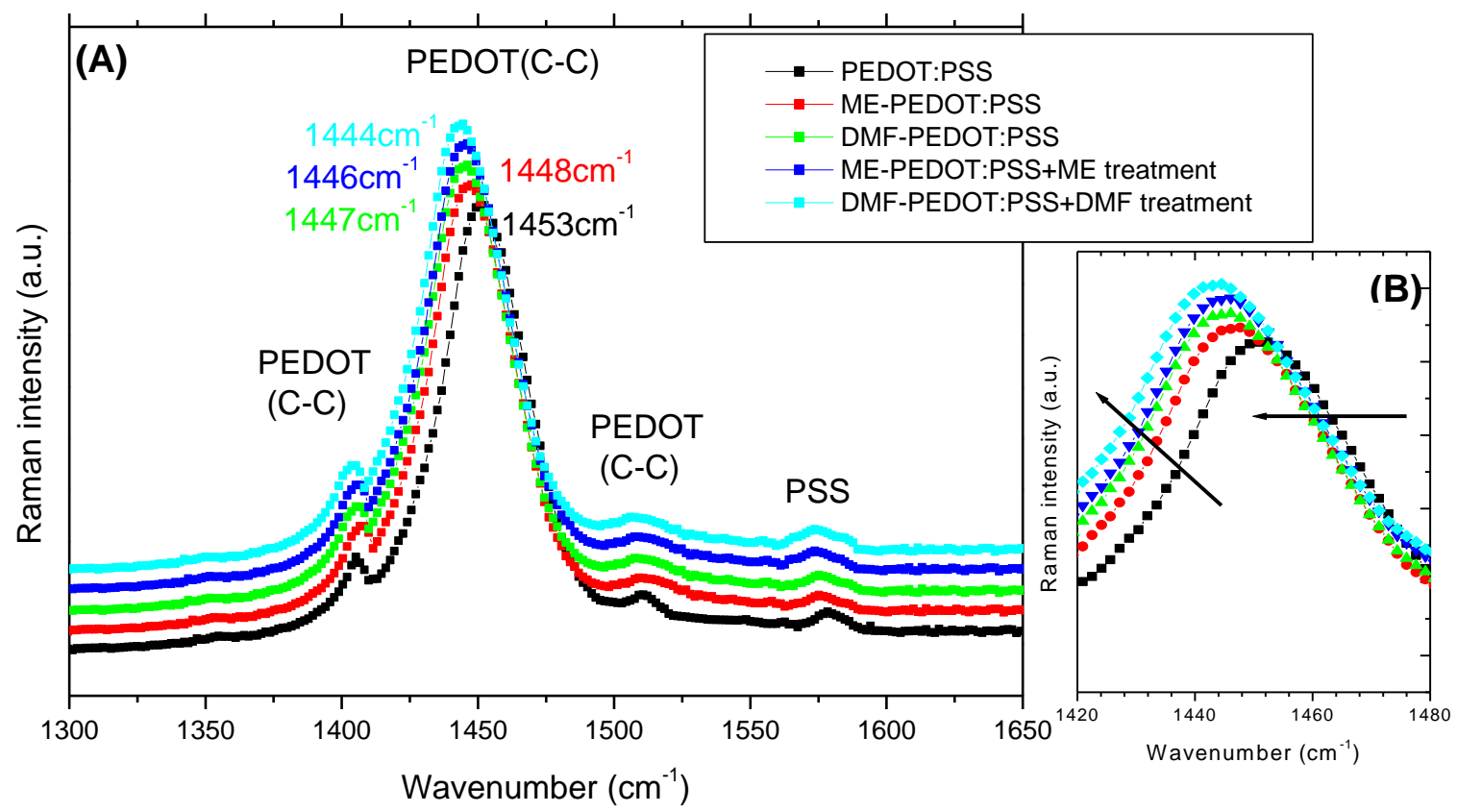

Fig.5; (A) Raman spectra for the studied PEDOT:PSS with and without treatment and $(B)$ the maximum peak around $1450 \mathrm{~cm}^{-1}$ 

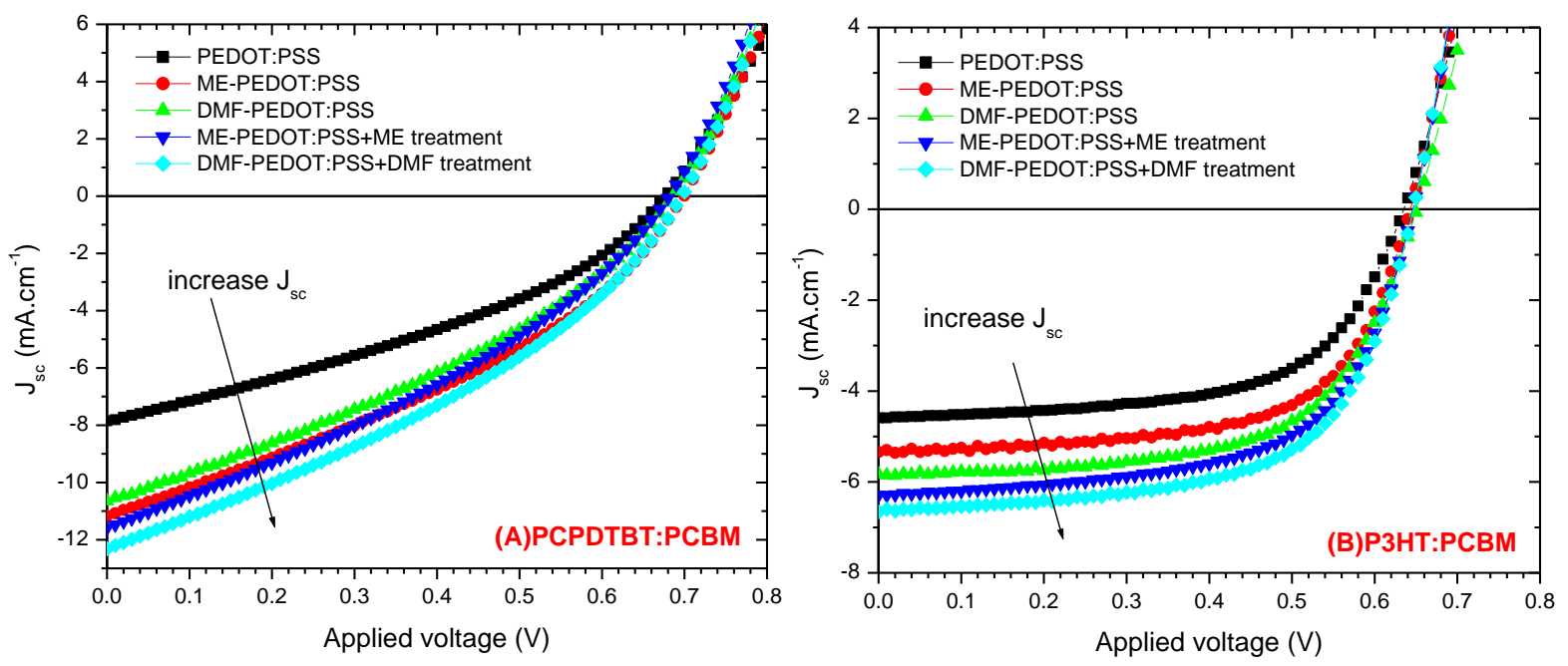

Fig.6; J-V characteristics of the (A) PCPDTBT:PCBM and (B) P3HT:PCBM based solar cells

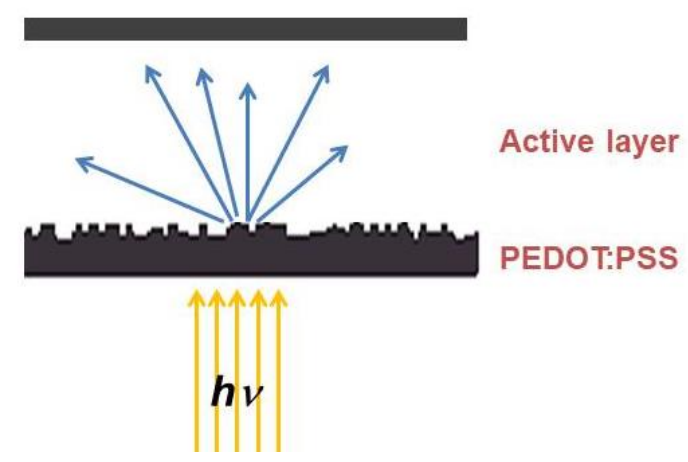

Fig.7; the assumption of light scattering from the rough PEDOT:PSS layer 

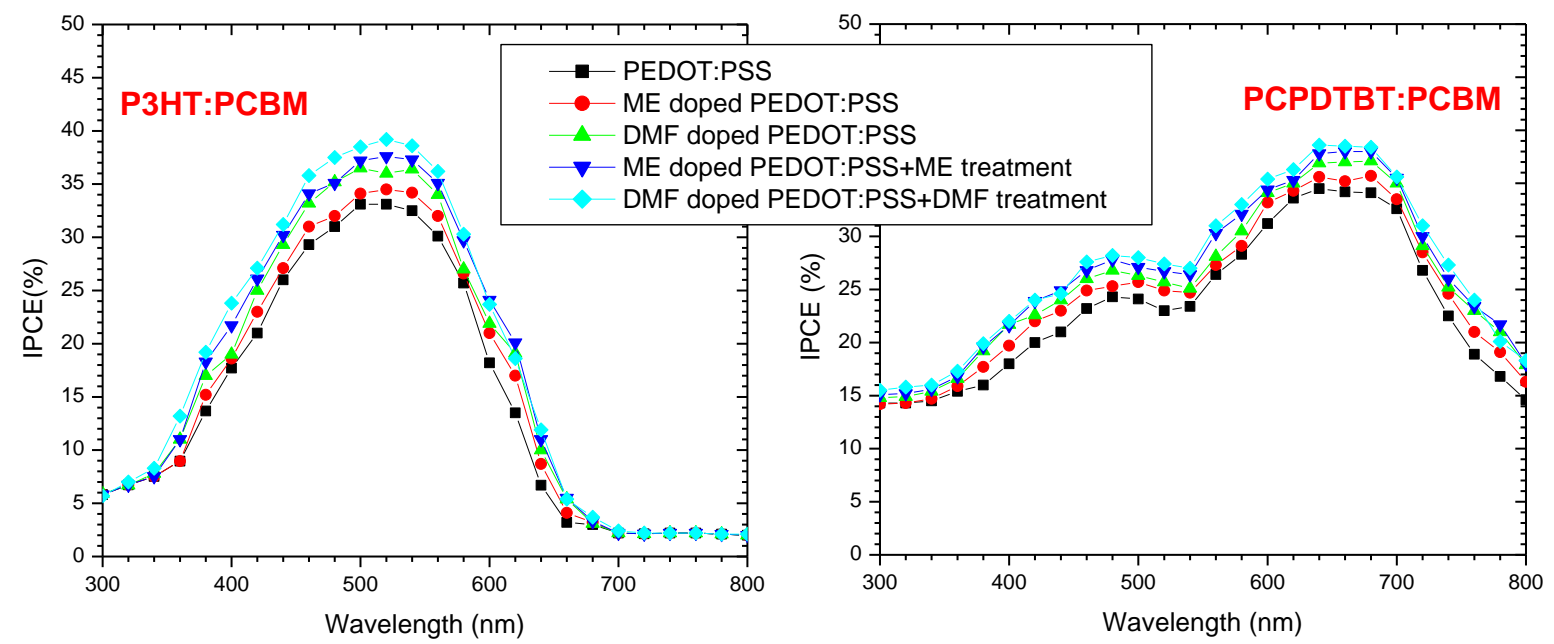

Fig.8: IPCE spectra for the PCPDTBT:PCBM and P3HT:PCBM based devices

Table 1; Photovoltaic properties of PCPDTBT:PCBM-based solar cells

\begin{tabular}{|c|c|c|c|c|c|}
\hline \multirow{2}{*}{ PCPDTBT:PCBM } & \multirow{2}{*}{ Pure } & \multirow{2}{*}{ ME } & \multirow{2}{*}{ DMF } & \multicolumn{2}{|c|}{ With solvent treatment } \\
\cline { 5 - 6 } & & & & ME & DMF \\
\hline $\mathrm{J}_{\mathrm{SC}}\left(\mathrm{mA} . \mathrm{cm}^{-2}\right)$ & 7.85 & 11.17 & 10.65 & 11.6 & 12.3 \\
\hline $\mathrm{V}_{\mathrm{OC}}(\mathrm{V})$ & 0.67 & 0.68 & 0.68 & 0.68 & 0.69 \\
\hline $\mathrm{FF}$ & 0.36 & 0.36 & 0.34 & 0.34 & 0.35 \\
\hline PCE $\%$ & 1.87 & 2.7 & 2.47 & 2.63 & 2.93 \\
\hline $\mathrm{R}_{\mathrm{S}}(\Omega)$ & 98 & 94 & 92 & 86 & 87 \\
\hline
\end{tabular}

Table 2; Photovoltaic properties of P3HT:PCBM-based solar cells

\begin{tabular}{|c|c|c|c|c|c|}
\hline \multirow{2}{*}{ P3HT:PCBM } & \multirow{2}{*}{ Pure } & \multirow{2}{*}{ ME } & \multirow{2}{*}{ DMF } & \multicolumn{2}{|c|}{ With solvent treatment } \\
\cline { 5 - 6 } & & & & ME & DMF \\
\hline $\mathrm{J}_{\mathrm{SC}}\left(\mathrm{mA} . \mathrm{cm}^{-2}\right)$ & 4.59 & 5.38 & 5.88 & 6.31 & 6.63 \\
\hline $\mathrm{V}_{\mathrm{OC}}(\mathrm{V})$ & 0.63 & 0.64 & 0.65 & 0.64 & 0.64 \\
\hline $\mathrm{FF}$ & 0.61 & 0.62 & 0.63 & 0.64 & 0.66 \\
\hline $\mathrm{PCE} \%$ & 1.77 & 2.13 & 2.43 & 2.58 & 2.79 \\
\hline $\mathrm{R}_{\mathrm{S}}(\Omega)$ & 67 & 62 & 61 & 60 & 58 \\
\hline
\end{tabular}


مجلة أبحاث ميسان ، المجلد السادس عشر ، العدد الواحد والثلاثون ،حزيران ، سنة 2020 